\title{
Lumped Element Electrical Model based on Three Resistors for Electrical Impedance in Radiofrequency Cardiac Ablation: Estimations from Ana- lytical Calculations and Clinical Data
}

\author{
Enrique Berjano ${ }^{1, *}$ and Andre d'Avila ${ }^{2}$ \\ ${ }^{1}$ Biomedical Synergy, Electronic Engineering Department, Universitat Politècnica de València, Spain \\ ${ }^{2}$ Helmsley Cardiac Electrophysiology Center, Mt Sinai Medical Center and School of Medicine, New York, NY, USA
}

\begin{abstract}
The electrical impedance measured during radiofrequency cardiac ablation (RFCA) is widely used in clinical studies to predict the heating evolution and hence the success of the procedure. We hypothesized that a model based on three resistors in series can mimic the total electrical impedance measured during RFCA. The three resistors or impedances are given by: impedance associated with the tissue around the active electrode (myocardium and circulating blood) (Z-A), that associated with the tissue around the dispersive electrode (Z-DE) and that associated with the rest of the body (Z-B). Our objective was to quantify the values associated with these three impedance types by an analytical method, after which the values obtained would be compared to those estimated from clinical data from previous studies. The results suggest that an RFCA using a 7 Fr 4-mm electrode would give a Z-A of around 75 ohms, a Z-DE around 20 ohms, and Z$B$ would be $15 \pm 10 \mathrm{ohms}$ (for body surface area variations between 1.5 and $2.5 \mathrm{~m}^{\wedge} 2$ ). Finally, adaptations of the proposed model were used to explain the results of previous clinical studies using a different electrode arrangement, such as in bipolar ablation of the ventricular septum.
\end{abstract}

Keywords: Cardiac ablation, electrical impedance, lumped element model, percutaneous ablation, radiofrequency ablation, theoretical model, three-resistor model.

\section{INTRODUCTION}

Radiofrequency (RF) cardiac ablation (RFCA) is a procedure employed to eliminate cardiac arrhythmias [1]. An electrode placed at the tip of a percutaneous catheter is used to identify the target and then RF voltage is applied between this (active) electrode and a large patch (dispersive, indifferent or return electrode) placed on the patient's skin. The applied voltage produces an electrical current between both electrodes (Fig. 1). The very high electrical current density in the tissue in contact with the active electrode causes localized necrosis by electrocoagulation. This is really a monopolar electrosurgical procedure in the form of electrocoagulation based on a high value of electrical current (1-2 A), low voltage $(<200 \mathrm{~V})$ and good contact between the active electrode and tissue, which means low electrical impedance $(Z)$. From a macro point of view, the $Z$ measured during RFCA is the ratio between the applied voltage and flowing current. From a micro point of view, $Z$ is the result of taking into account the values of the electrical resistivity $(\rho)$ of each micro-portion of tissue through which electrical current passes. Consequently, the electrical resistivity (inverse of electrical conductivity, $\sigma$ ) is a micro-type electrical characteristic, whereas the electrical impedance is macro-type. In

*Address correspondence to this author at the Electronic Engineering Department (Building 7F), Universitat Politècnica de València, Camino de Vera, 46022 Valencia, Spain; Tel: 34-963877607; Fax: 34-963877609; E-mail: eberjano@eln.upv.es
RF ablation, tissue can be considered as purely resistive, i.e. the capacitive component (e.g. due to the cell membranes) of the impedance is negligible compared to the resistive component, hence impedance and resistance are equivalent terms in this context. All this is also true for other ablation procedures, such as those conducted to treat tumors by focal RF ablation.

Previous modeling studies used numerical models (e.g. based on the Finite Element Method) to explore electrical and thermal behavior during RFCA [2]. In this approach, the physical domain of the model (electrodes and biological tissue) is divided into small elements in which the electrical

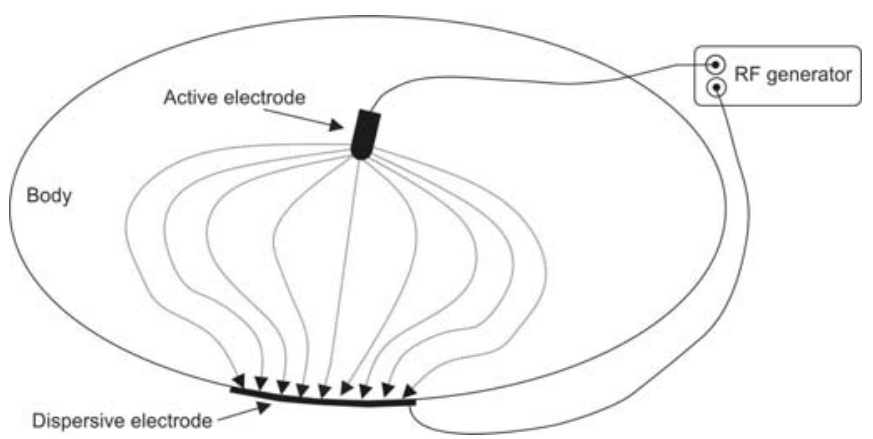

Fig. (1). In radiofrequency catheter ablation, electrical current flows between an active electrode and a dispersive electrode placed on the patient's skin. 
(Laplace's equation) and thermal (bioheat equation) governing equations are solved [2]. By setting the electrical conductivity $\sigma$ for each small element, the numerical problem involves obtaining total impedance $Z$, which is the sum of the contributions of all the elements. This type of model is known in engineering circles as the distributed element model, as the attributes (e.g. electrical resistance) are distributed evenly throughout the material (biological tissue). In theory, all the elements are infinitely small and are electrically connected to each other in an ideal way, i.e. with no electrical resistance.

In contrast, the lumped element models widely used in engineering assume that the attributes are lumped in the domain. In the case of RFCA, a distributed model has the advantage of computing the distribution of electrical current throughout the biological tissue, which may not be uniform. The benefits of lumped element models consist of their simplicity and usefulness for teaching purposes. For the RFCA case, we hypothesized that a lumped element model based on three resistors in series could mimic the total electrical impedance measured during the process. The three resistors, or impedances, would be associated with (i) the tissue around the active electrode (myocardium and circulating blood), (ii) tissue around the dispersive electrode, and (iii) the rest of the body. Our objective was to quantify the values associated with these three types of impedance by an analytical method, after which the values obtained would be compared to others estimated from clinical data obtained from previous studies. We considered this model could be used to explain in a simple way the electrical and thermal behavior of specific arrangements in RFCA, such as using two dispersive electrodes, constant current instead of constant voltage, or a bipolar system.

\section{ARRANGEMENT OF THE MODEL}

By considering that the tissue has a homogeneous rectangular prism shape with an electrical resistivity $\rho$, such as that shown in Fig. (2), the impedance could be estimated as follows:

$$
Z=\rho \cdot \frac{L}{A}
$$

where $L$ is the total length and $A$ the (cross-section) area through which electrical current flows. Equation (1) shows an essential concept: the electrical resistivity $\rho$ depends on the tissue type, while the impedance depends not only on electrical resistivity (i.e. tissue type) but also on the geometric parameters (length traveled by the current and the crosssection of the path). By taking the idea of the rectangular prism into account (which is obviously an oversimplification), we can assume that the tissue surrounding the active electrode can be divided into concentric spherical shells of identical thickness $L$ and areas $\left(A_{1}<A_{2}<A_{3} \ldots\right)$ that increase with distance from the electrode (see Fig. 3). This graphical interpretation was proposed by Wittkampf and Nakagawa [3]. The area $A$ through which electrical current flows can be called the effective area (cross-section) and the shells are in fact rectangular prisms. Then, using Equation (1) and assuming that tissue is homogeneous (i.e. all spherical shells have the same electrical resistivity), the tissue can be considered

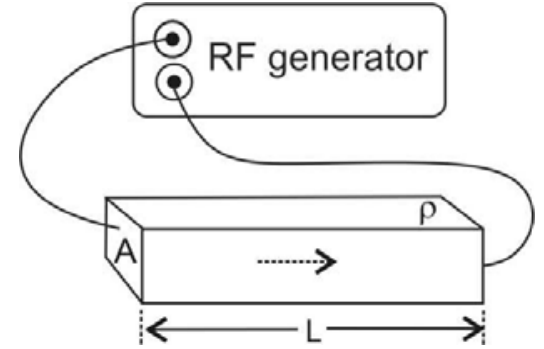

Fig. (2). If we simplify and consider a homogeneous tissue with a shape of rectangular prism, the impedance measured by the RF generator is directly proportional to the length $\mathrm{L}$ of the tissue, directly proportional to the electrical resistivity $\rho$, and inversely proportional to the area A through which electrical current flows (dashed arrow).

as a series of fragments with areas that increase with distance from the electrode, i.e. with decreasing impedance values $\left(Z_{1}>Z_{2}>Z_{3} \ldots\right)$. The impedance measured by the RF generator (total impedance) is therefore the sum of the individual impedance of each tissue fragment $\left(Z=Z_{1}+Z_{2}+Z_{3} \ldots\right)$. In practical terms, this means that the tissue next to the active electrode contributes more to the measured impedance than tissue further away. Another possible explanation is that the current density distribution shows a decreasing gradient with distance from the electrode. Now we can extend this model based on surfaces with identical thickness $L$ to all the paths of the RF electrical currents (see Fig. 3). Intuitively we can identify three zones comprised of prisms of different areas:

1. Tissue around the active electrode, which is associated with very small effective areas and hence contributes a high proportion of total impedance $\left(Z_{A}\right)$.

2. Tissue of the body through which electrical current flows, at this level mainly through paths (well perfused organs and large vessels) [4] which offer little resistance, i.e. the effective areas are large $\left(Z_{B}\right)$.

3. Tissue around the dispersive electrode which is associated with effective areas slightly smaller than those inside the body but much larger than those near the active electrode $\left(Z_{D E}\right)$.

The identification of these three zones is thus used as the basis of the proposed three-resistor model, as shown in Fig. (4). The objective of this study was to somehow quantify the values associated with these three types of impedance (resistors), which was achieved by using data reported from previous RFCA clinical studies. As far as we are aware that this is the first three-resistors-in-series RF ablation model simulating the active electrode, body and dispersive electrode. We tried to estimate the three impedance values in two different ways: firstly by using analytical calculations and then indirectly from values reported in clinical studies.

\section{ANALYTICAL ESTIMATE OF THE THREE RESIS- TOR VALUES}

\section{Resistance Associated with the Tissue Around the Active Electrode (ZA)}

Ragheb et al. [5] proposed that the electrical impedance of a spherical monopolar electrode totally surrounded by a 


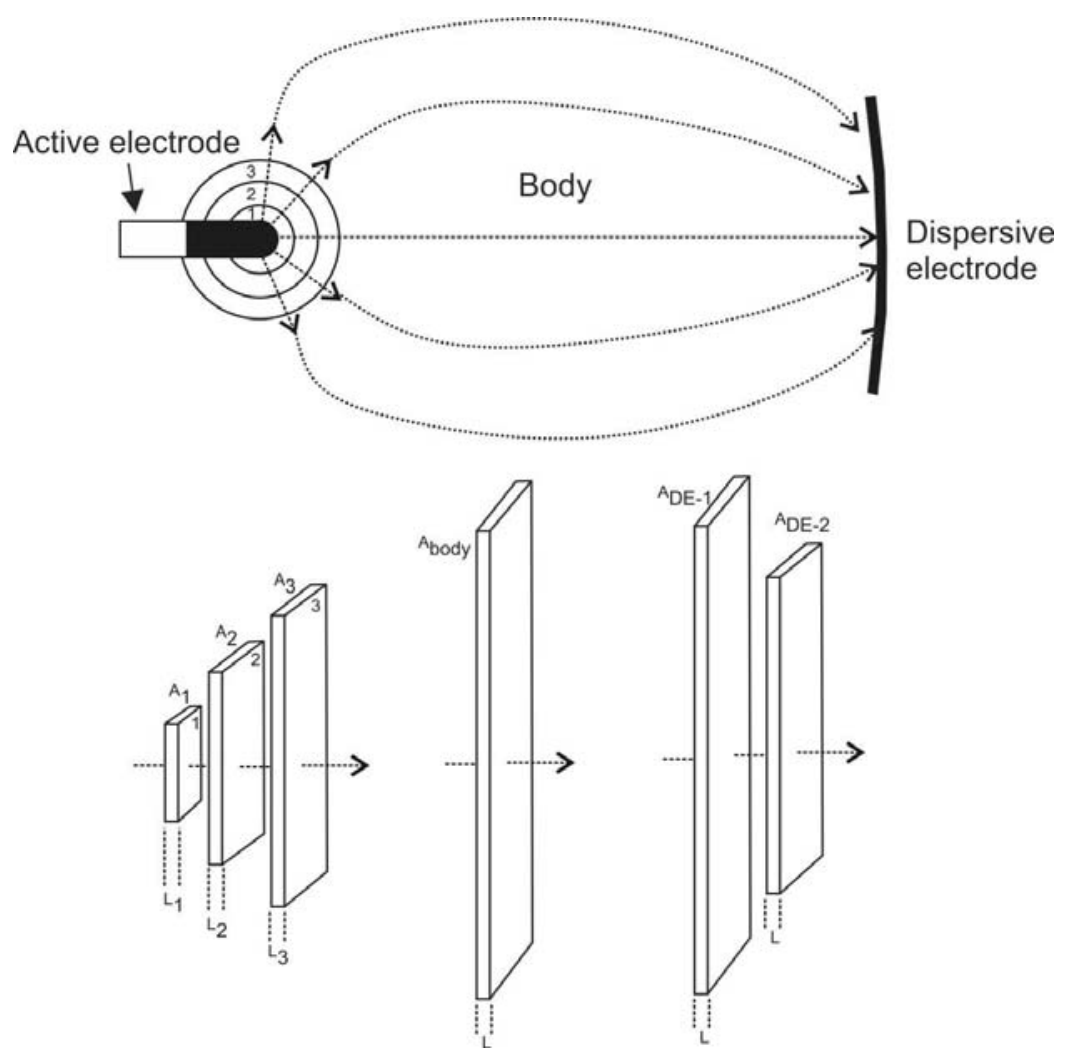

Fig. (3). The tissue surrounding the active electrode can be divided into concentric spherical shells with identical thickness L, and increasing area $\left(\mathrm{A}_{1}<\mathrm{A}_{2}<\mathrm{A}_{3} \ldots\right)$ as we move away from the electrode. At the same time, all the tissue in the path of the RF current can be divided into surfaces with identical thickness $\mathrm{L}$, and increasing area $\left(\mathrm{A}_{1}<\mathrm{A}_{2}<\mathrm{A}_{3} \ldots\right)$ as we move away from the active electrode. Inside the body, i.e. far away from the active and dispersive electrodes, the current flows across a large area $\left(\mathrm{A}_{\text {body }}\right)$. In the proximity of the dispersive electrode the areas $\left(\mathrm{A}_{\mathrm{DE}}\right)$ are slightly smaller, but are always larger than those of the tissue next to the active electrode.

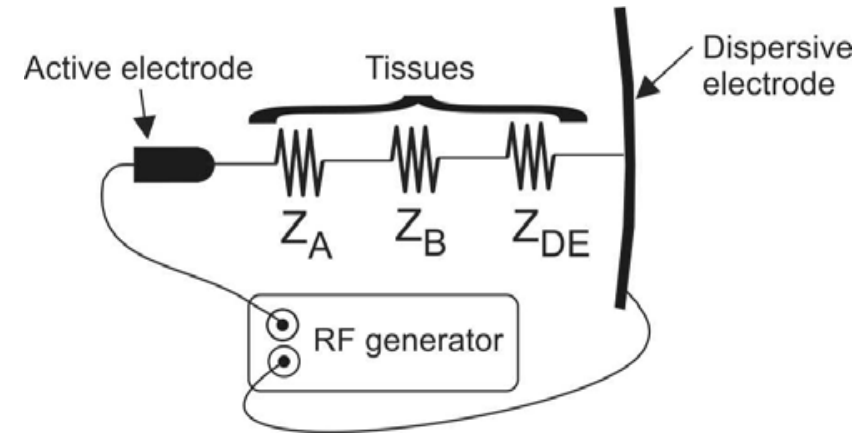

Fig. (4).Three-resistor electrical model for $R F$ cardiac ablation. The aim is to quantify the values of the three impedances (resistors $Z_{A}, Z_{B}$ and $\mathrm{Z}_{\mathrm{DE}}$ ) connected in series, which represent the total impedance measured by the RF generator.

conducting medium (saline) is the series combination of two impedances: electrode-electrolyte interface impedance and the impedance of the medium itself. Their experimental results showed that the former is negligible for frequencies above $100 \mathrm{kHz}$, which means that for electrosurgical frequencies as used in RFCA $(300 \mathrm{kHz}-1 \mathrm{MHz})$ only the impedance of the medium need be considered as contributing to $Z_{A}$.

In order to estimate the value of $Z_{A}$ of the model in the present study, we considered the case of a standard $7 \mathrm{Fr}$ diameter and $4 \mathrm{~mm}$ long catheter electrode, comprised of a cylindrical prism with a semispherical tip and a total surface of $27 \mathrm{~mm}^{2}$. Although previous computer modeling studies have shown that current density near the active electrode is not homogeneous [6], here we considered that the distribution is uniform and so assumed a spherical active electrode for the estimate. The radius of an effective area of $27 \mathrm{~mm}^{2}$ of a spherical electrode is $1.47 \mathrm{~mm}$. We also assumed the active electrode to be surrounded by a homogeneous medium. Under these conditions the impedance (resistance) can be estimated as follows [5]:

$$
Z=\frac{1}{4 \pi a \sigma}
$$

where $a$ is the electrode radius. The electrical conductivity $\sigma$ of the tissues depends on several factors, but in the context of the RFCA temperature and tissue type can be considered the most significant. Tissue type has a strong influence on 
conductivity, e.g. fat $(0.02 \mathrm{~S} / \mathrm{m})$ is much lower than cardiac tissue $(0.6 \mathrm{~S} / \mathrm{m})$, and blood $(0.8 \mathrm{~S} / \mathrm{m})$ [7]. Temperature is also important as conductivity normally increases by around $+2 \% /{ }^{\circ} \mathrm{C}$. Since heating is mainly confined to the tissue next to the active electrode, the variation in its conductivity is mostly responsible for the change in electrical impedance.

The relative position of the active electrode and cardiac wall in RFCA has two limiting cases: A) poor electrode contact with the cardiac wall, i.e. almost floating in the cardiac chamber and totally surrounded by blood; and B) firm electrode contact, i.e. completely inserted into the cardiac wall. Using Equation (2) with an electrode radius $a=1.47$ $\mathrm{mm}$ gives a $Z_{A}$ estimate of $90 \Omega$ in case $\mathrm{B}$ and $67 \Omega$ in case A, i.e. a difference of $23 \Omega$.

The usual situation during RFCA is an active electrode partially inserted into the tissue with its surface partially in contact with the cardiac tissue and the remainder surrounded by circulating blood. The impedance associated with the tissue around the active electrode consequently ranged between 67 and $90 \Omega$. Fig. (5) shows a modification of the three-resistor electrical model in which the impedance associated with the active electrode is in fact the parallel association of two impedances, which correspond to the current passing through the tissue $\left(Z_{A-\text { tissue }}\right)$ and that through the blood $\left(Z_{A-b l o o d}\right)$. Due to the high cooling power of circulating blood, only the tissue is heated and hence only $Z_{A-t i s s u e}$, varies during heating. Fig. (6) shows resistance variation during heating in a typical RFCA. It can be seen that the drop in impedance during $\operatorname{RFCA}(\Delta Z)$ is almost entirely due to variations in $Z_{\text {A-tissue }}$.
If different areas of active electrode are considered, e.g. 2.5 and $8 \mathrm{~mm}$ long instead of $4 \mathrm{~mm}$, the area will be around $18 \mathrm{~mm}^{2}$ (equivalent to a $1.19 \mathrm{~mm}$ radius spherical electrode) and $57 \mathrm{~mm}^{2}$ (equivalent to a $2.13 \mathrm{~mm}$ radius spherical electrode) respectively. As a consequence, $Z_{A}$ will be between 83 and $111 \Omega$ for a $2.5 \mathrm{~mm}$ electrode and 46 and $62 \Omega$ for an 8 $\mathrm{mm}$ electrode. In other words, using an 8 - $\mathrm{mm}$ electrode instead of a 7-mm electrode would reduce $Z_{A}$ by between 21 and $28 \Omega$, according to the insertion depth of the active electrode into the tissue. Likewise, using a smaller electrode of $2.5 \mathrm{~mm}$ instead of 4-mm would increase $Z_{A}$ by between 16 and $21 \Omega$, according to the insertion depth.

\section{Resistance Associated with the Tissue Next to the Disper- sive Electrode (ZDE)}

Dispersive electrodes with an effective area of around $150 \mathrm{~cm}^{2}$ (also known as plates, patches, ground electrodes or reference electrodes) are normally used in monopolar electrosurgical procedures. $Z_{D E}$ impedance is due to the tissue in contact with the dispersive electrode, i.e. skin and muscle. Pearce [8] suggested values ranging between 2 and $40 \Omega$, according to electrode design and skin preparation.

The value of $Z_{D E}$ can be estimated analytically by using Yamamoto and Yamamoto's proposal [9] of dividing this impedance into two series resistances $R_{k}$ and $R_{c}$ so that $Z_{D E}$ will be the sum of $R_{k}+R_{c}$. The former is associated with the outer layer of the skin (keratin content) and can be modeled as:

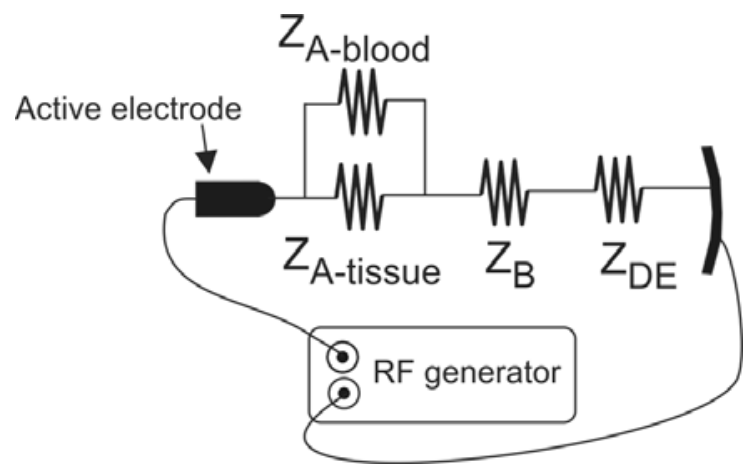

Fig. (5). Modification of the three-resistor electrical model shown in Fig. (4) with a combination of two parallel resistors associated with the distal electrode: cardiac tissue impedance $\left(\mathrm{Z}_{\mathrm{A} \text {-tissue }}\right)$ and blood impedance $\left(\mathrm{Z}_{\mathrm{A} \text {-blood }}\right)$.

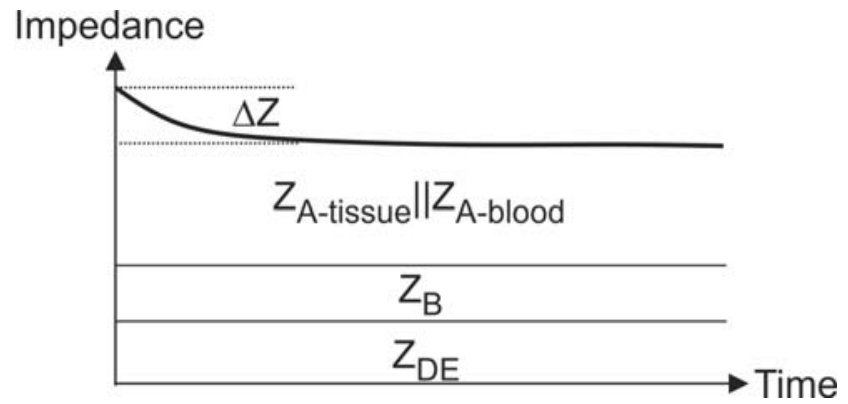

Fig. (6). Typical impedance evolution (thick line) during radiofrequency cardiac ablation. This impedance is the sum of a three-resistors-inseries combination: impedance associated with the dispersive electrode $\left(\mathrm{Z}_{\mathrm{DE}}\right)$, that associated with the body $\left(\mathrm{Z}_{\mathrm{B}}\right)$, and the parallel combination of cardiac tissue $\left(\mathrm{Z}_{\mathrm{A} \text {-tissue }}\right)$ and blood impedance $\left(\mathrm{Z}_{\mathrm{A} \text {-blood }}\right)$. Only $\mathrm{Z}_{\mathrm{A} \text {-tissue }}$ varies during the ablation, due to heating at the target, and hence the decrease of total impedance $(\Delta Z)$ is exclusively due to this component. 
$R_{k}=\frac{\rho_{k} d}{S}$

where $\rho_{k}$ is the average tissue resistivity $\left(10^{2} \Omega \cdot \mathrm{m}\right.$ at 500 $\mathrm{kHz}$, Miklavcic et al. 2006)[10], $S$ is electrode area and $d$ is the thickness of this layer $(40 \mu \mathrm{m})$. If a dispersive electrode area of $150 \mathrm{~cm}^{2}$ is considered, then $R_{k} \approx 0.25 \Omega$.

The latter, the so-called spreading resistance, is associated with deeper tissues and is calculated as the resistance between infinity and a circular plate electrode on a semiinfinite medium as follows [11]:

$$
R_{c}=\frac{\rho_{C}}{4 d}
$$

where $\rho_{c}$ is the average tissue resistivity and $d$ is electrode diameter. A value of $3.3 \Omega \cdot \mathrm{m}$ was considered for $\rho_{c}$, which has been reported for the low-lying layers of skin at $500 \mathrm{kHz}$ [10]. If a dispersive electrode area of $150 \mathrm{~cm}^{2}$ is considered and we simplify the usual rectangular shape of dispersive electrodes to a circular shape, this modeled electrode would have a diameter of $\approx 14 \mathrm{~cm}$ and from Equation (4) we estimate $R_{c} \approx 6 \Omega$. The value of $Z_{D E}$ from these analytical calculations would thus be around $6 \Omega$.

\section{Resistance Associated with the Body (ZB)}

The value of $Z_{B}$ is difficult to estimate analytically since the body is highly heterogeneous from an electrical point of view. Some tissues have high conductivity (e.g. wellperfused organs) and are the favored paths of electrical currents, while others such as lung and fat tissues are poor conductors. Although Pearce [8] suggested a range between 20 and $60 \Omega$ for $Z_{B}$, no direct analytical calculations can be carried out. If we consider a mean $Z_{B}$ value of $40 \Omega$, total impedance $\left(Z_{A}+Z_{B}+Z_{D E}\right)$ estimated analytically for a 4-mm $7 \mathrm{Fr}$ electrode would be between 113 and $136 \Omega$, according to the quality of the electrode/tissue contact.

\section{ESTIMATE FROM CLINICAL DATA}

The real situation inside the tissues during RFCA is actually based on distributed elements. However, here we hypothesized that a lumped-element model comprising three resistors could explain some of the results reported in previ- ous clinical studies. Numerous RFCA clinical studies on 4$\mathrm{mm}$ (7 Fr) electrodes have reported impedance values around $100 \Omega$, e.g. $113 \pm 10 \Omega$ in 45 patients [12], $103.5 \pm 8.6 \Omega$ in 76 patients [13], $100 \pm 9 \Omega$ in 29 patients [14].

Our analytical estimate ranged from 113 to $136 \Omega$ in terms of total impedance with a 4-mm $7 \mathrm{Fr}$ electrode, which is somewhat higher than those measured clinically and is probably due to the $40 \Omega Z_{B}$ value we selected from a general electrosurgical procedure. We also think that it is probably lower in the case of RFCA. The following subsections attempt to estimate $Z_{A}$ and $Z_{D E}$ and, indirectly, the $Z_{B}$ values from previous results of clinical RFCA studies and to compare them with those obtained analytically.

\section{Resistance Values Associated with the Tissue Around the Active Electrode (ZA)}

The $Z_{A}$ values obtained from analytical estimates for the cases of very poor contact (electrode almost floating in cardiac chamber) and firm contact (electrode completely inserted into the cardiac wall) were of $67 \Omega$ and $90 \Omega$ respectively, i.e. a difference of $23 \Omega$. This value is closely in agreement with that found by Strickberger et al. [15] in a clinical study conducted on 25 patients using a 7 Fr catheter and $4 \mathrm{~mm}$-long electrode. They found that impedance was $25 \pm 15 \Omega$ higher when the catheter was firmly in contact rather than in poor contact with the right ventricle. This value also agrees with that found by Cao et al. [16] in an ex vivo experimental study in which the difference between a tip electrode inserted $6 \mathrm{~mm}$ into the endocardium (firm contact) and a depth of $-4 \mathrm{~mm}$ (poor contact) at $500 \mathrm{kHz}$ was around $30 \Omega$.

In a study on five patients, Wittkampf and Nakagawa [3] recently attempted to quantify the impedance associated with the tissue next to the active electrode $Z_{A}$ (which they called interface impedance) and the remainder impedance, which corresponds to $Z_{B}+Z_{D E}$ in our model, which they termed rest of the patient. They introduced two 4-mm electrodes into the patient and then took three measurements: two from each electrode individually and a third from the two electrodes connected in parallel. Fig. (7A) shows an adaptation of the 3resistor model to this case. By assuming that the $Z_{A}$ associated with each electrode $\left(Z_{A-1}\right.$ and $\left.Z_{A-2}\right)$ was almost identical,

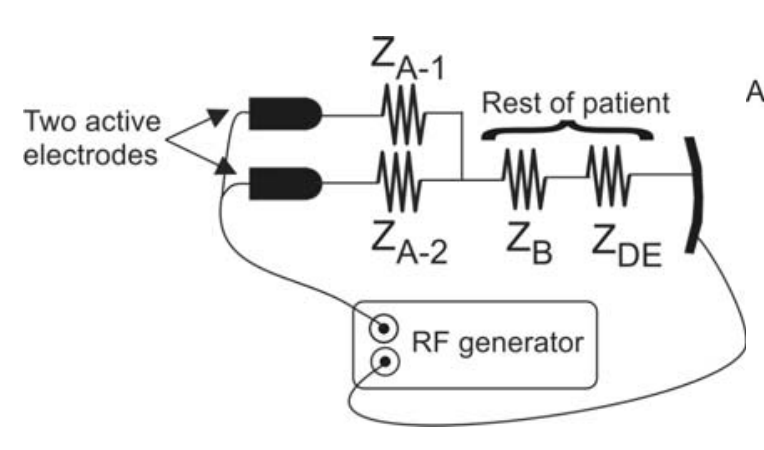

A

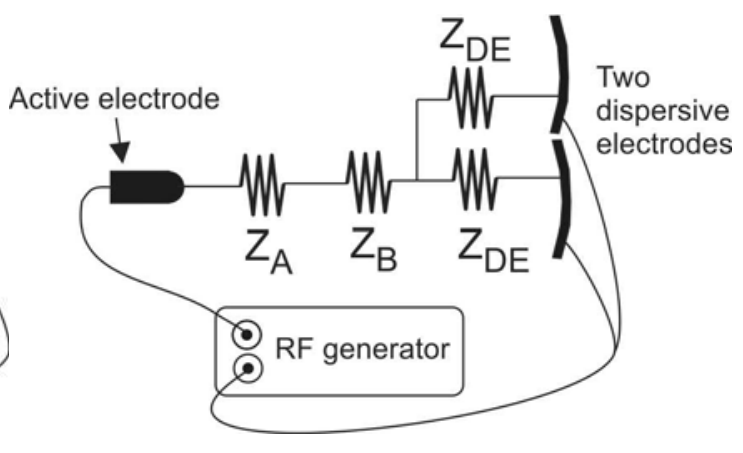

B

Fig. (7). A: Resistor model adapted to the case of RFCA with two active electrodes. B: Resistor model adapted to the case of RFCA with two dispersive electrodes. All these models assume that $\mathrm{Z}_{\mathrm{A}-1}, \mathrm{Z}_{\mathrm{A}-2}$, and $\mathrm{Z}_{\mathrm{A}}$ are the parallel combination of the cardiac tissue impedance $\left(\mathrm{Z}_{\mathrm{A}-\mathrm{tissue}}\right)$ and blood impedance $\left(\mathrm{Z}_{\mathrm{A}-\mathrm{blood}}\right)$, as detailed in Fig. (5). 
they were able to estimate a value for $Z_{A}$ of $75 \pm 11 \Omega$, which coincides with the range proposed in our model (67-90 $\Omega$ ). They were also able to estimate a value for the rest of the patients of $Z_{B}+Z_{D E}=47 \pm 11 \Omega$.

We also used clinical data from previous studies on electrodes of different lengths to check the validity of the $Z_{A}$ values estimated analytically, obviously assuming that the change in the active electrode surface affects only $Z_{A}$ though not $Z_{B}$ or $Z_{D E}$. Rodriguez et al. [17] measured the initial impedances in 15 patients using a 4-mm electrode and in another 15 patients by an $8-\mathrm{mm}$ electrode. They found the $4-$ $\mathrm{mm}$ gave $97 \pm 7 \Omega$ and the 8 -mm $72 \pm 4 \Omega$, i.e. a mean difference of $25 \Omega$. This closely agrees with our analytical estimate, which suggests a difference of between 21 and $28 \Omega$, according to the insertion depth of the active electrode. The difference in impedance found by Sacher et al. [18] with 4$\mathrm{mm}$-irrigated- electrodes $(116 \pm 12 \Omega)$ and 8 -mm electrodes $(92 \pm 15 \Omega)$ is also inside this estimated range $(24 \Omega)$.

Jackman et al. [19] found an impedance value of $103 \pm 22$ $\Omega$ for a $4-\mathrm{mm}$ and $148 \pm 40 \Omega$ for a 2.5 -mm electrode, which gives a mean difference of $45 \Omega$. This value is higher than our analytical estimates (16-21 $\Omega$ ), probably due to finer catheters being used on some patients ( $6 \mathrm{Fr}$ instead of $7 \mathrm{Fr}$ ), which would give a higher impedance value than the estimate.

\section{Values for the Resistance Associated with the Tissue Around the Dispersive Electrode (ZDE)}

We estimated the value of $Z_{D E}$ to be around $6 \Omega$. Nath et al. [20] studied the effect of the area of the dispersive electrode on recorded total impedance in 20 patients by a $4-\mathrm{mm}$ $7 \mathrm{Fr}$ electrode with the dispersive electrode $\left(139.5 \mathrm{~cm}^{2}\right)$ between the scapulae and a second electrode on the left thigh. This arrangement gave a significantly lower baseline impedance ( $95 \pm 6 \Omega$ vs. $101 \pm 10 \Omega$ ) and allowed them to estimate a value for $Z_{D E}$. The double dispersive electrode arrangement could be similar to the situation shown in Fig. (7B), in which the impedances associated with the tissue in contact with the dispersive electrodes are in parallel. In this case, the measured impedance would be:

$$
Z=Z_{D}+Z_{B}+Z_{D E} \| Z_{D E}
$$

The value of two identical impedances in parallel is equal to half of one of the values. Since Nath et al. [20] did not find any significant differences in the baseline impedance $(101 \pm 10 \Omega$ vs. $101 \pm 9 \Omega)$ of the two electrode positions, we can assume that both $Z_{D E}$ are comparable. The value of $Z_{D E}$ would therefore be twice the difference between the cases, i.e. $2 \times(101-95 \Omega)=12 \Omega$. The same group [20] also found that the difference between the single and double dispersive electrode arrangement was more marked in the nine patients with $>100 \Omega$ baseline impedance: $110 \pm 6 \Omega$ vs. $100 \pm 4 \Omega$. With these results the estimated value of $Z_{D E}$ would be $2 \times(110-100 \Omega)=20 \Omega$.

Santoro et al. [21] conducted another clinical study on eleven patients with a $7 \mathrm{Fr}-4 \mathrm{~mm}$ electrode to examine the effect of area (single vs. multiple dispersive electrodes) on measured impedance. They found that impedance was significantly lower for single patches placed on the anterior or posterior chest than for those placed on the lateral chest or buttock. They also found impedance to be significantly lower for combinations of two or more patches. By using the impedances measured with a single patch on the anterior $(108 \pm 8 \Omega)$ and posteromedial $(107 \pm 8 \Omega)$ chest measured with a double arrangement $(99 \pm 8 \Omega)$ we can estimate a new $Z_{D E}$ value of $2 \times(108-99 \Omega)=18 \Omega$.

As these $Z_{D E}$ values estimated from clinical data differ from the analytically estimated values $(6 \Omega)$, we consider it likely that $R_{k}$ can be highly influenced by the skin preparation procedure used.

\section{Values for the Resistance Associated with the Body (ZB)}

We first considered a range between 20 and $60 \Omega$ for $Z_{B}$, as suggested by Pearce [8]. No direct analytical calculations can be carried out for this value. After reviewing the mean values of total impedance reported in previous clinical studies using a 4-mm (7 Fr) electrode (around $100 \Omega$ ) we could estimate $Z_{B}$ as:

$Z_{B}=Z-Z_{A}-Z_{D E}$

If we consider the impedance values reported in clinical studies between 100 [12] and $113 \Omega$ [11], then the estimated value of $Z_{B}$ would be approximately $110-75-20=15 \Omega$. Also, it is known that the value of total impedance in RFCA can be influenced by the body surface area [22-24]. We may also assume that body surface area (BSA) would affect only $Z_{B}$ and not $Z_{A}$ and $Z_{D E}$, i.e. any change in total impedance $(\Delta Z)$ will be caused by a change in $Z_{B}\left(\Delta Z_{B}\right)$. Borganelli et al. [22] found an increase of around $20 \Omega$ (from 90 to $110 \Omega$ ) in patients with BSA between 1.5 and $2.5 \mathrm{~m}^{2}$. On the other hand for patients with a BSA $<1.5 \mathrm{~m}^{2}$ Park et al. [23] found no correlation between $Z$ and BSA, so that as patient size falls below $1.5 \mathrm{~m}^{2}$, impedance remains constant. We hypothesized that this constant would have a value of approximately the sum of $Z_{A}+Z_{D E}+Z_{B \min }$ (see Fig. 8).

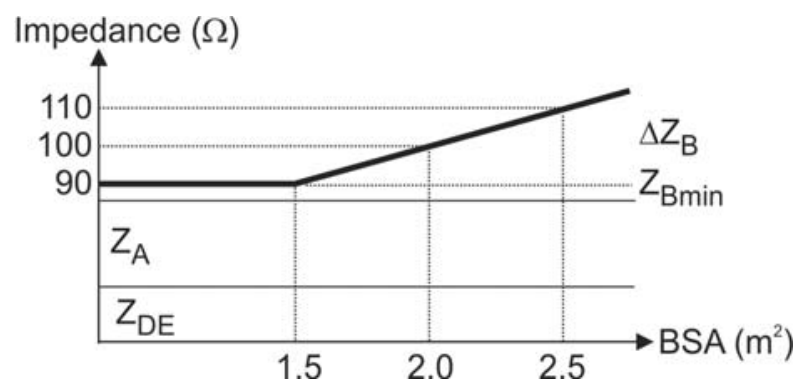

Fig. (8). Total impedance separated into its constituent parts in relation to body surface area. 
COMPLETE MODEL AND PROPOSAL OF RESISTOR VALUES

The above data suggest that for an RFCA using a 4-mm 7 Fr electrode:

$Z_{A}$ could have a value around $75 \Omega$, similar to that obtained by Wittkampf and Nakagawa [15], ranging from 67 to $90 \Omega$ for cases of poor electrode/tissue contact, and completely surrounded by cardiac tissue, respectively.

1. $Z_{D E}$ could have a value around $20 \Omega$. We consider that the analytically estimated value $(6 \Omega)$ is highly influenced by the skin preparation and is much lower than those obtained from clinical studies.

2. $Z_{B}$ could be around $15 \Omega$ for a BSA of $2.0 \mathrm{~m}^{2}, \pm 10 \Omega$ for a variation of $\pm 0.5 \mathrm{~m}^{2}$ in BSA, and a constant value for $\mathrm{BSA}<1.5 \mathrm{~m}^{2}$.

3. To sum up, for a $Z_{A}=75 \Omega$ and $Z_{D E}=20 \Omega, Z_{B}=15 \pm 10$ $\Omega$ (for a change of BSA between 1.5 and $2.5 \mathrm{~m}^{2}$ ).

\section{CLINICAL IMPLICATIONS}

A distributed element, such as previous theoretical models based on the Finite Element Method (FEM), assumes that tissue electrical conductivity is evenly distributed throughout the tissue. However, we propose here a lumped-element model which assumes that electrical conductivity is lumped into three resistors joined by perfectly conducting wires. In a distributed model each tissue fragment is considered to be infinitesimally small. As no wires are perfect conductors, this is really analogous to a transmission line model, since the electrical characteristic is provided as $\mathrm{S} / \mathrm{m}$ (i.e. Siemens per length unit).

We are aware that the lumped model based on three resistors is clearly inaccurate when compared to an FEM model when studying, for instance, temperature distributions around the active electrode. In spite of this limitation we believe that the lumped-element model proposed here can explain some of the results found in past and future clinical studies and especially provide a simple tool to understand the concept of electrical impedance in RFCA. The following subsection addresses some of these issues.

\section{Constant Current vs. Constant Voltage Ablation}

We can first consider that the thermal effect on the tissue (lesion creation at the target) depends exclusively on the power dissipated in the cardiac tissue $\left(Z_{A \text {-tissue }}\right)$, since the $R F$ current passing through the body, the tissue beneath the dispersive electrode and circulating blood do not cause perceptible heating. The power transformed into heat in the target zone would thus be proportional to $P_{A}=I^{2} Z_{A}$, where $I$ is the total current flowing between active and dispersive electrodes. In this way, the proposed model clarifies some of the conclusions obtained in previous clinical studies in which different ways of delivering RF power were used (constant voltage and constant current). For instance, one such study reported on the effect of varying electrode dispersive area using constant power or constant current [21]. They found that lesion size was independent of the dispersive electrode area when a constant current was used and that higher power was necessary to achieve the same lesion size. These results can be perfectly explained by the proposed model; the increased area of the dispersive electrodes implies a decrease in $Z_{D E}$, which only affects the power dissipated at this zone $\left(I^{2} Z_{D E}\right)$ but does not affect the lesion created, which depends on $I^{2} Z_{A}$. In addition, with constant current, the electrode dispersive area and the impedance associated with the body affect power requirements due to:

$$
P_{\text {TOTAL }}=I^{2} Z_{A}+I^{2} Z_{B}+I^{2} Z_{D E}
$$

If we are using constant current, $P_{T O T A L}=I^{2}\left(Z_{A}+Z_{B}+Z_{D E}\right)$ and hence required total power is proportional to the impedances of each zone. The greater the dispersive electrode area the lower $Z_{D E}$ and hence power requirements are also lower.

On the other hand, ablation with constant voltage $(V)$ means that the lesion size will be influenced by the dispersive electrode area, since the power transformed into heat in the target zone is proportional to the total current $I, P_{A}=I^{2} Z_{A}$, and this current is inversely proportional to the sum of impedances, I.

$$
I=\frac{V}{Z_{A}+Z_{B}+Z_{D E}}
$$

\section{Bipolar Ablation of Ventricular Septum}

Although RFCA employs electrical current in a monopolar mode (between an active electrode and a grounding pad), in order to increase lesion size investigators have recently proposed bipolar RF ablation by using a pair of electrodes located on each side of the ventricular septum [25]. For this case we can adjust the three-resistor model to mimic this situation in terms of electrical impedance.

The impedance associated with the tissue included in a 7 $\mathrm{mm}$ radius shell around the active electrode (which is approximately half the septum thickness of $15 \mathrm{~mm}$ ) represents almost $90 \%$ of the total impedance associated with the active electrode given by Equation (2). This means that in a bipolar $\mathrm{RF}$ ablation on a $15 \mathrm{~mm}$ thick septum we could assume a model of two resistors, such as that shown in Fig. (9), in

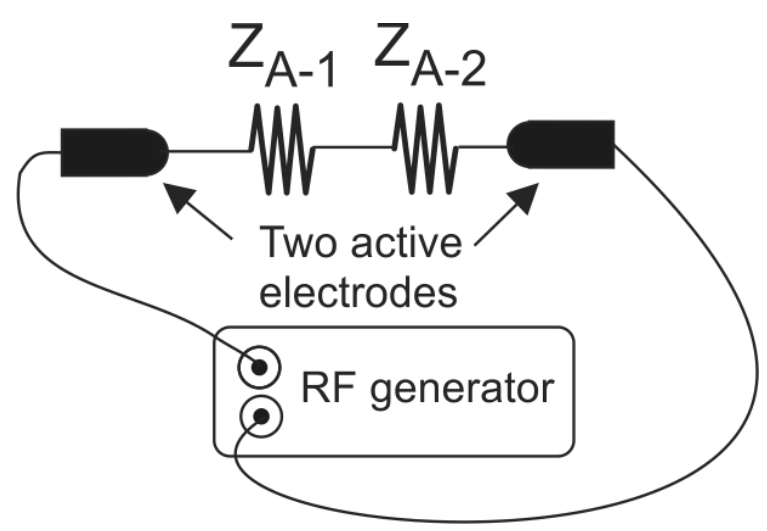

Fig. (9). Resistor model adapted to the case of bipolar RFCA on the septum. No dispersive electrode is used here. This model assumes that each $\mathrm{Z}_{\mathrm{A}-1}$ and $\mathrm{Z}_{\mathrm{A}-2}$ are also the parallel combinations of the impedances of the cardiac tissue $\left(\mathrm{Z}_{\mathrm{A} \text {-tissue }}\right)$ and blood $\left(\mathrm{Z}_{\mathrm{A} \text {-blood }}\right)$, as detailed in Fig. (5). 
which each resistor $\left(Z_{A-1}\right.$ and $\left.Z_{A-2}\right)$ corresponds to the impedance of the tissue around each electrode. The values for $Z_{B}$ and $Z_{D E}$ in this case are zero. By keeping in mind the role of $Z_{A}$ in the drop in impedance during heating (see Fig. 6) it is clear that in the case of a bipolar ablation of the septum the drop will be about twice that in a monopolar ablation, since both $Z_{A-1}$ and $Z_{A-2}$ contribute to the reduced impedance. This has been accurately observed in an in vitro study by comparing the impedance drop in the case of bipolar $(32.5 \pm 14.5 \Omega$ ) and monopolar modes (15.8 $\pm 5.8 \Omega$ ) [25].

Finally, it should be pointed out that in general the values of the proposed model were obtained using dry electrodes. Other designs, such as irrigated (closed or open) electrodes could give different $Z_{A}$ values, but this does not invalidate the rationale of the three-resistor model.

Future work could compare the estimated values of the three resistors with those obtained from distributed element models such as Finite Element Models. Other clinical data could also be used to refine the range of the proposed values of the three impedances, or to particularize them to specific conditions.

\section{CONCLUSIONS}

The results suggest that for an RFCA using a $7 \mathrm{Fr} 4-\mathrm{mm}$ electrode, $Z_{A}$ would be around $75 \Omega, Z_{D E}$ around $20 \Omega$, and $Z_{B}$ would be $15 \pm 10 \Omega$ (for a BSA range between 1.5 and 2.5 $\mathrm{m}^{2}$ ). Finally, adaptations of the proposed model also help to explain the results of previous clinical studies using different electrode arrangements, such as bipolar ablation of the cardiac septum.

\section{CONFLICT OF INTEREST}

The authors confirm that this article content has no conflicts of interest.

\section{ACKNOWLEDGEMENTS}

This work received financial support from the Spanish "Plan Nacional de I+D+I del Ministerio de Ciencia e Innovación" Grant No. TEC2011-27133-C02-01.

\section{REFERENCES}

[1] S. Nath, and D. E. Haines, "Biophysics and pathology of catheter energy delivery systems", Prog. Cardiovasc. Dis., vol. 37, pp. 185204, January-February 1995.

[2] E. J. Berjano, "Theoretical modeling for radiofrequency ablation: state-of-the-art and challenges for the future", Biomed. Eng. Online., vol. 5, p. 2, April 2006

[3] F. H. Wittkampf, and H. Nakagawa, "RF catheter ablation: Lessons on lesions", Pacing Clin. Electrophysiol., vol. 29, pp. 1285-1297, November 2006

[4] G. R. Neufeld GR, "Principles and hazards of electrosurgery including laparoscopy", Surg. Gynecol. Obstet., vol. 147, pp. 705710, November 1978.

[5] T. Ragheb, S. Riegle, L. A. Geddes, and V. Amin, "The impedance of a spherical monopolar electrode" Ann. Biomed. Eng., vol. 20, pp. 617-627, 1992.

[6] D. Panescu, J. G. Whayne, S. D. Fleischman, M. S. Mirotznik, D. K. Swanson, and J. G. Webster, "Three-dimensional finite element analysis of current density and temperature distributions during radio-frequency ablation", IEEE Trans. Biomed. Eng., vol. 42, pp. 879-890, September 1995.
[7] K. R. Foster, and H. P. Schwan, "Dielectric properties of tissues and biological materials: a critical review", Crit. Rev. Biomed. Eng., vol. 17, pp. 25-104, 1989.

[8] J. A. Pearce, Electrosurgery Chapman and Hall: London, 1986.

[9] T. Yamamoto, and Y. Yamamoto, "Electrical properties of the epidermal stratum corneum", Med. Biol. Eng., vol. 14, pp. 151-158, March 1976.

[10] D. Miklavcic, N. Pavselj, and F. X. Hart, "Electric Properties of Tissues" In: Wiley Encyclopedia of Biomedical Engineering, M. Akay, Ed. Wiley: Hoboken, 2006, pp. 1-14.

[11] M. Saito, K. Nakayama, M. Hori, and Y. Fujimori, "A fundamental study on the electrodes for cardiac pacemakers", Jpn. J. Med. Electron. Biol. Eng., vol. 5, pp. 192-198, 1967.

[12] E. Nsah, R. Berger, L. Rosenthal, R. Hui, B. Ramza, P. Jumrussirikul, J. H. Lawrence, G. Tomaselli, D. Kass, and H. Calkins, "Relation between impedance and electrode temperature during radiofrequency catheter ablation of accessory pathways and atrioventricular nodal reentrant tachycardia", Am. Heart J., vol. 136, pp. 844-851, November 1998

[13] Z. C. Wen, S. A. Chen, C. E. Chiang, C. T. Tai, S. H. Lee, Y. Z. Chen, W. C. Yu, J. L. Huang, and M. S. Chang, "Temperature and impedance monitoring during radiofrequency catheter ablation of slow AV node pathway in patients with atrioventricular node reentrant tachycardia", Int. J. Cardiol., vol. 57, pp. 257-263, December 1996.

[14] S. A. Strickberger, J. Hummel, M. Gallagher, C. Hasse, K. C. Man, B. Williamson, V. R. Vorperian, S. J. Kalbfleisch, F. Morady, and J. J. Langberg, "Effect of accessory pathway location on the efficiency of heating during radiofrequency catheter ablation", $\mathrm{Am}$. Heart J., vol. 129, pp. 54-58, January 1995.

[15] S. A. Strickberger, V. R. Vorperian, K. C. Man, B. D. Williamson, S. J. Kalbfleisch, C. Hasse, F. Morady, and J. J. Langberg, "Relation between impedance and endocardial contact during radiofrequency catheter ablation", Am. Heart J., vol. 128, pp. 226-229, August 1994.

[16] H. Cao, S. Tungitkusolmun, Y. B. Choy, J. Z. Tsai, V. R. Vorperian, and J. G. Webster, "Using electrical impedance to predict catheter-endocardial contact during RF cardiac ablation", IEEE Trans. Biomed. Eng., vol. 49, pp. 247-243, March 2002.

[17] L. M. Rodriguez, A. Nabar, C. Timmermans, and H. J. Wellens, "Comparison of results of an 8-mm split-tip versus a 4-mm tip ablation catheter to perform radiofrequency ablation of type I atrial flutter", Am. J. Cardiol., vol. 85, pp. 109-112, January 2000.

[18] F. Sacher F, M. D. O'Neill, P. Jais, L. L. Huffer, J. Laborderie, N. Derval, A. Deplagne, Y. Takahashi, A. Jonnson, M. Hocini, J. Clementy, and M. Haissaguerre, "Prospective randomized comparison of 8-mm gold-tip, externally irrigated-tip and 8-mm platinum-iridium tip catheters for cavotricuspid isthmus ablation", $J$. Cardiovasc. Electrophysiol., vol. 18, pp. 709-713, July 2007.

[19] W. M. Jackman, X. Z. Wang, K. J. Friday, D. M. Fitzgerald, C. Roman, K. Moulton, P. D. Margolis, A. J. Bowman, K. H. Kuck, G. V. Naccarelli, J. V. Pitha, J. Dyer, and R. Lazzara, "Catheter ablation of atrioventricular junction using radiofrequency current in 17 patients. Comparison of standard and large-tip catheter electrodes", Circulation, vol. 83, pp. 1562-1576, May 1991.

[20] S. Nath, J. P. DiMarco, R. G. Gallop, I. D. McRury, and D. E. Haines, "Effects of dispersive electrode position and surface area on electrical parameters and temperature during radiofrequency catheter ablation", Am. J. Cardiol., vol. 77, pp. 765-767, April 1996.

[21] I. Santoro, W. Xunzhang, J. McClelland, K. Beckman, J. Oren, M. Arruda, C. Roman, H. Nakagawa, K. Hirao, R. Lazzara, and W. Jackman, "Effect of skin-patch location and surface area on impedance during radiofrequency catheter ablation", Pacing Clin. Electrophysiol., vol. 15, p. 580, 1992.

[22] M. Borganelli, R. el-Atassi, A. Leon, S. J. Kalbfleisch, H. Calkins, F. Morady, and J. J. Langberg, "Determinants of impedance during radiofrequency catheter ablation in humans", Am. J. Cardiol., vol. 69, pp. 1095-1097, April 1992.

[23] J. K. Park, B. D. Halperin, J. Kron, S. R. Holcomb, and M. J. Silka, "Analysis of body surface area as a determinant of impedance during radiofrequency catheter ablation in adults and children", $J$. Electrocardiol., vol. 27, pp. 329-332, October 1994.

[24] D. Wang, J. E. Hulse, E. P. Walsh, and J. P. Saul, "Factors influencing impedance during radiofrequency ablation in humans", Chin. Med. J. (Engl), vol. 108, pp. 450-455, June 1995. 
[25] J. S. Koruth, S. Dukkipati, M. A. Miller, P. Neuzil, A. d'Avila, and V. Y. Reddy, "Bipolar irrigated radiofrequency ablation: a therapeutic option for refractory intramural atrial and ventricular tachy- cardia circuits", Heart Rhythm, vol. 9, pp. 1932-1941, December 2012.

Received: March 05, 2013

Revised: April 26, 2013

Accepted: May 23, 2013

(C) Berjano and d'Avila; Licensee Bentham Open.

This is an open access article licensed under the terms of the Creative Commons Attribution Non-Commercial License (http://creativecommons.org/licenses/by-nc/3.0/) which permits unrestricted, non-commercial use, distribution and reproduction in any medium, provided the work is properly cited. 